\title{
LATERAL PAIN IN AN ATHLETE'S KNEE: A RARE CASE OF DISLOCATION OF THE FEMORAL BICEPS TENDON
}

Aires Duarte Junior' ${ }^{1}$ Nilson Severino ${ }^{2}$, Ana Paula Simões da Silva ${ }^{3}$, Marcos Vaz de Lima ${ }^{3}$, Vanessa Ribeiro Resende ${ }^{4}$, Paulo F. Kertzman ${ }^{4}$

\section{ABSTRACT}

Dislocation of the femoral biceps tendon is rare and is described clinically in the literature as a lateral pain in the knee. It was initially reported as an anomalous insertion of the long head of the femoral biceps. Subsequently, it was found to be caused by abnormal mobility of the tendon over the prominence of the fibular head at certain angles of knee flexion. The objective of the present report was to describe and discuss a condition of lateral

\section{INTRODUCTION}

Subluxation of the biceps femoris tendon is rare and has been scantly described in the literature. The etiology and treatment are controversial in the few articles found $^{(1-8)}$. Anatomic changes of the proximal fibular prominence or frequent microlesions due to sprains are believed to occur ${ }^{(1)}$, but even in these cases, intraoperative findings have clearly shown an anomalous insertion of the tendon of the biceps femoris.

Anatomical variations of the complex involving the biceps femoris tendon insertion have recently been described $^{(7)}$ in a study describing the long and short head, each of which are divided into two different arms, and the long head has a tendon and aponeurotic component (Figure 1).

- The direct arm (DA) inserts in the posterolateral region of the fibular head and the anterior arm (AA) inserts in the anterolateral region. The two arms of the tendon component insert adjacent to the tibia. knee pain in a swimmer who started to present subluxation of the femoral biceps during sports practice, which incapacitated him from taking part in trials and competitions. The case is discussed in the light of the literature surveyed; the likelihood that the etiology for the trauma leading to this condition was repetition; and the surgical treatment instituted, which led to excellent results and the patient's return to his habitual sports practice.

Keywords - Athletes, Femoral Biceps, Knee

- The aponeurotic arm of the long head inserts in the posterior region, forming the reflex arm (RA) and also expands in the insertion of the iliotibial tract (ITT), in Gerdy's tubercle.

We report an unusual case of dislocation of the biceps femoris likely resulting from repetitive trauma during swim training in an athlete without apparent anatomical anomalies.

\section{CASE REPORT}

A male patient of 19 years, who swims competitively, reports training four hours a day every day of the week. He was doing fine when, after routine training, he perceived a click on the lateral region of the left knee, initially without pain, and that did not hinder his usual activities. The onset of pain began after two months.

He was initially treated with physical therapy and after one month without improvement, reported a progressive increase in pain and inability to engage

1 - Head Physician, Sports Trauma Group, Santa Casa de São Paulo - São Paulo, SP, Brazil.

2 - Doctor's degree and Master's degree, Department of Orthopedics and Traumatology, School of Medical Sciences, Santa Casa de Misericórdia de São Paulo; Head Physician, Knee Group, Santa Casa de São Paulo - São Paulo, SP, Brazil.

3 - Master's degree; Assistant, Sports Trauma Group, Santa Casa de São Paulo - São Paulo, SP, Brazil.

4 - Master's degree student, Department of Orthopedics and Traumatology, School of Medical Sciences, Santa Casa de Misericórdia de São Paulo; Physician and Volunteer, Sports Trauma Group - São Paulo, SP, Brazil.

Study conducted at the Department of Orthopedics and Traumatology, School of Medical Sciences, Santa Casa de Misericórdia de São Paulo - SCMSP.

Correspondence: Pavilhão Fernandinho Simonsen - Santa Casa de Misericórdia de São Paulo. Rua Cesário Mota Júnior, 112, Santa Cecília - 01221-020 - São Paulo, SP. Email: apaulass@gmail.com

Received for publication: 12/5/2011, accepted for publication: 2/1/2012

The authors declare that there was no conflict of interest in conducting this work 


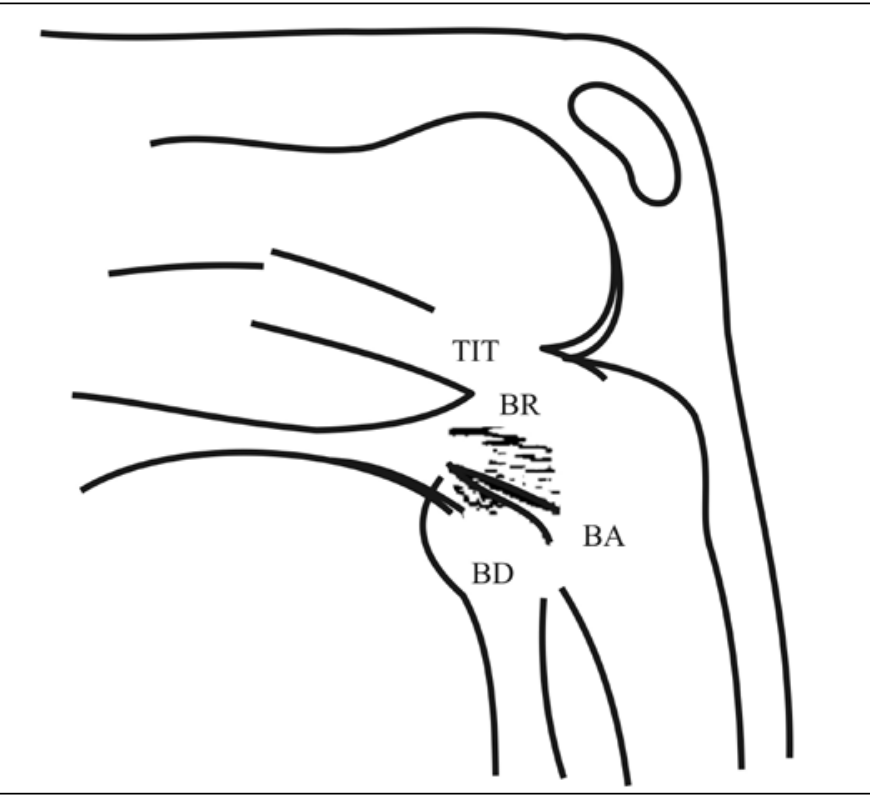

Figure 1 - Schematic representation of the insertion of the long head of the biceps femoris in the fibula AA: anterior arm + DA: direct arm = form the tendinous part of the long head. ITT: iliotibial tract + RA: reflex portion $=$ form an aponeurotic expansion of the long head of the biceps.

in sports and, despite the use of analgesics and antiinflammatory medication, was unable to ambulate normally. He was instructed to start resting the limb for two weeks while physiotherapy was intensified, but persisted with pain and a lateral knee click with any flexion movement.

Physical examination showed no change in the range of motion, limb alignment was normal, and tests for ligamentous and meniscal injury were negative. The patient presented with pain on palpation of the head of the fibula and during knee flexion at around 80 degrees, we noted a subluxation of the long portion of the biceps femoris muscle over the fibular head at which time the patient reported pain. The phenomenon was more intense with the leg in internal rotation. The patient had no complaints regarding his normal contralateral knee.

The radiographs were normal without any prominence of the fibular head (Figure 2). The dynamic ultrasound showed that the tendon of the biceps femoris was subluxated on the fibular head.

Bursitis was present in the insertion region of the long arm of the biceps in the fibular head in magnetic resonance imaging, without any other abnormality in the tendon and its insertion or in the fibular head (Figure 3).

We opted for surgical treatment, during which it was possible to reproduce the subluxation even after

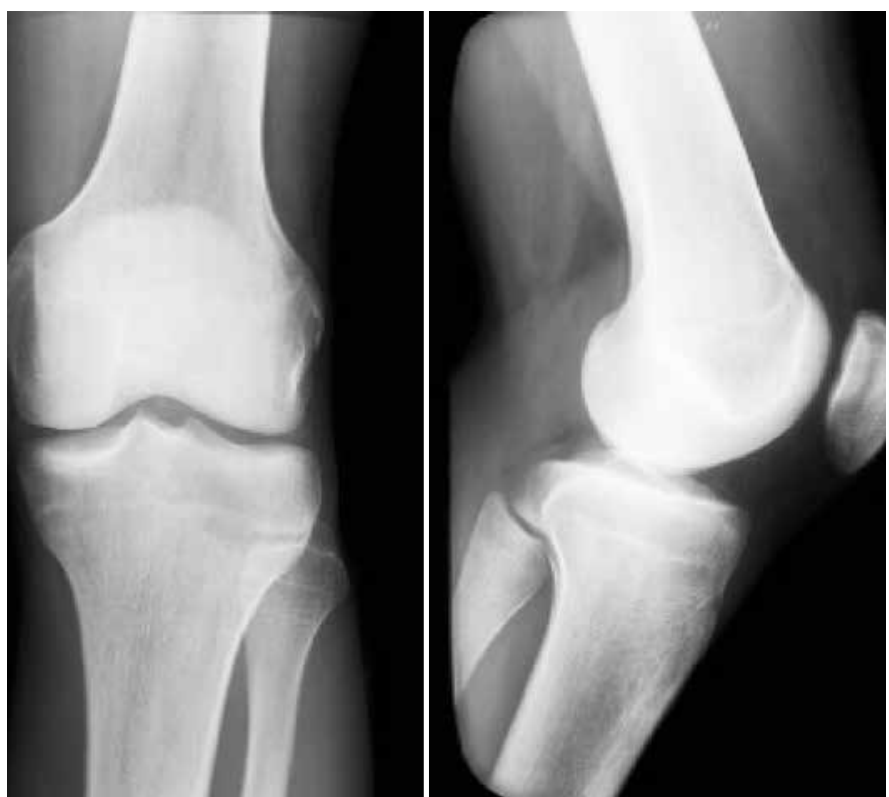

Figure 2 - Anteroposterior and lateral X-ray, without bone changes, of the knee affected by subluxation of the biceps femoris tendon.

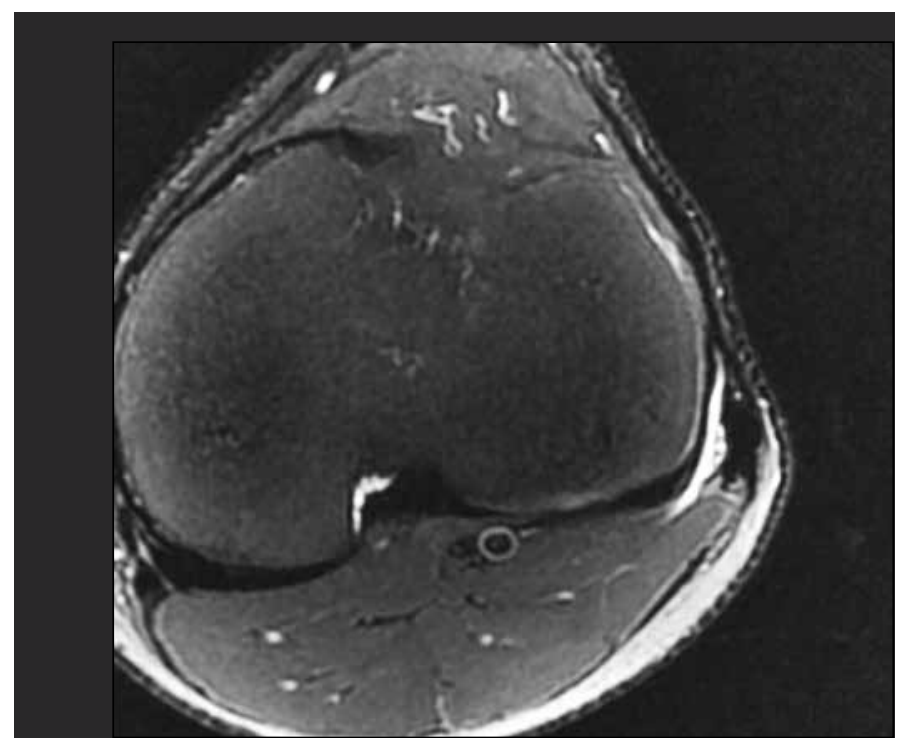

Figure $3-\mathrm{MRI}$ showing bursitis in the region at the insertion of the direct arm of the biceps femoris.

anesthesia, when it was above 80 degrees flexion with the leg in internal rotation. The insertion of the biceps was observed to be normal, but the tendon luxated on the fibular head during flexion. We performed osteoplasty of the fibular head, removing part of its lateral region (Figure 4). After this procedure, the tendon no longer clicked. No immobilization was used and full load was allowed in the immediate postoperative period.

The patient recovered well and, after 15 days, gradually returned to normal activity, reached a competitive level in two months, and is completely asymptomatic. 


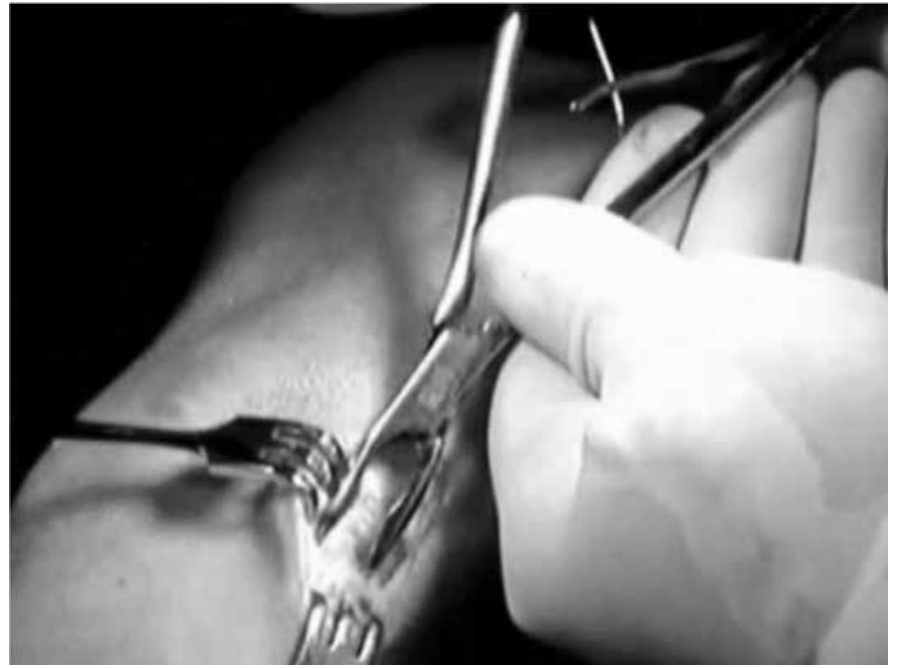

Figure 4 - Intraoperative and lateral osteoplasty of the fibular head.

\section{DISCUSSION}

The symptoms of knee "clicks", also called knee snapping, may be due to numerous causes both intra- and extra-articular. The intra-articular causes that are most commonly described are: discoid meniscus, meniscal injury, loose bodies, and synovial plica ${ }^{(2)}$. Extra-articular causes include iliotibial tract syndrome, popliteus tendon syndrome, semitendinosus syndrome and, more rarely, subluxation of the biceps femoris ${ }^{(1-8)}$.

The literature review shows eight reported cases ${ }^{(1-8)}$ of subluxation of the long portion of the biceps femoris - "snapping of the biceps femoris" - and, among them, several theories have been proposed to explain the origin of the disease. The complex and variable anatomy of the insertion of the tendon of the biceps femoris has been studied in great detail, the most recent study ${ }^{(8)}$ reveals that the long head of the biceps has a direct arm with insertion in the posterolateral region of the fibular head, and another anterior arm inserted in the anterolateral border of the fibular head adjacent to the tibia, both originating in the tendon. Another arm, the aponeurotic, called the reflex portion, inserts in the posterior border of the iliotibial tract and Gerdy's tubercle (Figure 1). In the case reported by Bansal et al. ${ }^{(7)}$, the authors suggest that the cause of the disease is the lesion of the reflex portion, as was found in our case. Table 1 shows the description and comparison of the cases reported in the literature.

Other authors ${ }^{(2,3,5,6)}$ suggest abnormal insertion of the long portion of the biceps, that is, more anterior on the fibular head. Other causes that have been suggested are more distal bifurcation of the long head of the biceps ${ }^{(4)}$ and prominence in the fibular head with normal insertion of the tendon ${ }^{(1)}$.

Six of the eight cases reported ${ }^{(1-6)}$ were bilateral (Table 1) and just two involved one limb, as in our case, and although most were bilateral, surgery was not always necessary on the contralateral side, which was asymptomatic. Only two cases were described as traumatic ${ }^{(7,8)}$.

In the case described, it was noted during surgery that the tendon of the long portion of the biceps femoris glided over the fibular head and the reflex portion was apparently ruptured, allowing slippage of the tendon that caused the click. Note that it is often difficult to characterize the etiology of dislocation because the use of a pneumatic tourniquet or even the muscle relaxation from anesthesia cause

Table 1 - Cases described in the global literature to the present date.

\begin{tabular}{|c|c|c|c|c|c|}
\hline Authors & Age/Sex & Trauma & Contralateral & Cause & Treatment \\
\hline Kristensen et al. ${ }^{(5)}$ & $20 / \mathrm{M}$ & no & yes & $\begin{array}{l}\text { Abnormal anterior insertion in the } \\
\text { tibia }\end{array}$ & Partial excision of the fibular head \\
\hline Lokiec et al. ${ }^{(6)}$ & 23/M & no & yes & $\begin{array}{l}\text { Abnormal anterior insertion in the } \\
\text { fibular head }\end{array}$ & Tendon reinserted posteriorly \\
\hline Hernandez et al..$^{(1)}$ & $16 / \mathrm{M}$ & yes & yes & $\begin{array}{l}\text { Abnormal anterior insertion in the } \\
\text { proximal tibia }\end{array}$ & $\begin{array}{l}\text { Suture of the tendon through a tunnel on the } \\
\text { fibular head }\end{array}$ \\
\hline $\begin{array}{l}\text { Kissenberth and } \\
\text { Wilckens }{ }^{(4)}\end{array}$ & 20/M & no & yes & $\begin{array}{l}\text { More distal bifurcation of the long } \\
\text { head of the biceps }\end{array}$ & Anterior arm cut and anchored posteriorly \\
\hline $\begin{array}{l}\text { Bach and } \\
\text { Minihane }^{(2)}\end{array}$ & 24/M & no & yes & $\begin{array}{l}\text { Fibular head prominence; normal } \\
\text { insertion }\end{array}$ & Partial bilateral excision of the fibular head \\
\hline $\begin{array}{l}\text { Bagchi and } \\
\text { Grelsamer }{ }^{(3)}\end{array}$ & 22/M & no & yes & $\begin{array}{c}\text { Abnormal anterior and proximal } \\
\text { insertion of the tibia }\end{array}$ & $\begin{array}{c}\text { Partial bilateral excision of the fibular head with } \\
\text { reinsertion }\end{array}$ \\
\hline Bansal et al. ${ }^{(7)}$ & 19/M & yes & no & Injury of the reflex portion & Resuture through a tunnel on the fibular head \\
\hline Our case & $19 / \mathrm{M}$ & no & no & Injury of the reflex portion & Lateral osteoplasty of the fibular head \\
\hline
\end{tabular}


subluxation to not occur. In this case, during the approach, synovial fluid output was noted after opening the bursa described on MRI, suggesting a local inflammatory process, and possibly confirming the presence of the local irritation process. We chose lateral osteoplasty in the fibular head. After this procedure, tendon stabilization was observed without requiring tenodesis.

Therefore, because subluxation of the long portion of the biceps femoris is a rare entity, with multiple causes, its surgical treatment is still controversial. Despite rigorous clinical examination, radiological investigation by magnetic resonance imaging and ultrasound, determining the etiology is difficult and often only clarified during surgery. In the literature, most cases do not have trauma as their initial cause. The description of anomalous and complex insertion of the tendon makes scheduling surgery difficult in symptomatic cases. Therefore, the decision of the best course of action should be made during surgery. Thus, knowledge of the local anatomy, of the probable causes, and of the different surgical techniques is essential to decide the best thing to be done during surgery.

\section{REFERENCES}

1. Hernandez JA, Ruis M, Noonan KJ. Snapping knee form anomalous biceps femoris tendon insertion: a case report. lowa Orthop J. 1996;16:161-3.

2. Bach BR, Minihane K. Subluxating biceps femoris tendon: an unusual case of lateral knee pain in a soccer athlete. Am J Sports Med. 2001;29(1); 93-5.

3. Bagchi K, Grelsamer RP. Partial fibular head resection for bilateral snapping biceps femoris tendon. Orthopedics. 2003;26(11);1146-9.

4. Kissenberth MJ, Wilckens JH. The snapping biceps femoris tendon. Am J Knee Surg. 2000;13(1):25-8.
5. Kristensen G, Nielsen K, Blyme PJ. Snapping knee form biceps femoris tendon. A case report. Acta Orthop. Scand. 1989;60(5):621.

6. Lokiec F, Velkes S, Shindler A, Pritsch M, The snapping biceps femoris syndrome. Clin Orthop Relat Res. 1992;(283):205-6.

7. Bansal R, Taylor C, Pimpalnerkar AL. Snapping knee: an unusual biceps femoris tendon injury. Knee. 2005;2(6):458-60.

8. Marshall JL, Girgis FG, Zelko RR. The biceps femoris tendon and its functional significance. J.Bone Joint Surg Am. 1972;54(7):1444-50. 